\title{
Effect of electron correlation on high-order-harmonic generation of helium atoms in intense laser fields: Time-dependent generalized pseudospectral approach in hyperspherical coordinates
}

\author{
Xiaoxu Guan, ${ }^{1}$ Xiao-Min Tong, ${ }^{2}$ and Shih-I Chu ${ }^{1}$ \\ ${ }^{1}$ Department of Chemistry, University of Kansas, and Kansas Center for Advanced Scientific Computing, Lawrence, Kansas 66045, USA \\ ${ }^{2}$ Institute of Materials Science, Graduate School of Pure and Applied Science, and Center for Computational Sciences, \\ University of Tsukuba, 1-1-1 Tennodai, Tsukuba, Ibaraki 305-8573, Japan
}

(Received 28 November 2005; published 2 February 2006)

\begin{abstract}
We present a time-dependent generalized pseudospectral (TDGPS) approach in hyperspherical coordinates for fully $a b$ initio nonperturbative treatment of multiphoton dynamics of atomic systems in intense laser fields. The procedure is applied to the investigation of high-order-harmonic generation (HHG) of helium atoms in ultrashort laser pulses at a $\mathrm{KrF}$ wavelength of $248.6 \mathrm{~nm}$. The six-dimensional coupled hypersphericaladiabatic-channel equations are discretized and solved efficiently and accurately by means of the TDGPS method. The effects of electron correlation and doubly excited states on HHG are explored in detail. A HHG peak with Fano line profile is identified which can be attributed to a broad resonance of doubly excited states. Comparison of the HHG spectra of the $a b$ initio two-electron and the single-active-electron model calculations is also presented.
\end{abstract}

DOI: 10.1103/PhysRevA.73.023403 PACS number(s): 42.50.Hz, 32.60. +i, 32.80.Rm, 32.80.Fb

\section{INTRODUCTION}

The study of multiphoton and very-high-order nonlinear optical processes in intense ultrashort laser pulses is a subject of much current interest and significance in science and technology [1]. In particular, multiple high-order-harmonic generation (HHG) is one of the most rapidly developing topics in strong-field atomic, molecular, and optical (AMO) physics in the past decade [2]. The generation of harmonics of orders in excess of 300 from rare-gas atoms has been recently demonstrated by experiments [3-5] using laser pulses shorter than 20 femtoseconds (fs) and peak intensity more than $10^{14} \mathrm{~W} / \mathrm{cm}^{2}$. Examples of the potential applications of the HHG include the development of coherent soft x-ray laser light source and nonlinear optics in the extreme ultraviolet (XUV) regime [1-5], attosecond laser pulses [6-8], comb laser technology [9-11], etc.

To study such intense-field HHG processes for $N$-electron atomic systems using the $a b$ initio wave function approach, it is necessary to solve the time-dependent Schrödinger equation of $3 N$-spatial dimensions, which is well beyond the capability of the current supercomputer technology for $N>2$. For the two-electron $(N=2)$ case, several approaches have been proposed [12], however, fully converged sixdimensional (6D) calculation is still difficult to achieve and remains a major computational challenge in the study of strong-field AMO physics today. One of the approximations commonly used for the treatment of strong-field processes in the past decade is the single-active-electron (SAE) model with frozen core [13]. The SAE approach has been successfully applied to the investigation of HHG of rare-gas atoms in linearly polarized laser fields, providing useful insights regarding strong-field atomic dynamics. However, within the SAE approach, the effects of electron correlation and doubly excited states and the role of the individual spin orbital cannot be explicitly treated. The recent development and application of the self-interaction-correction (SIC) time- dependent density functional theories (TDDFTs) have overcome most of these difficulties. These SIC-TDDFT methods provide powerful nonperturbative approaches for the treatment of multiphoton ionization (MPI) and HHG processes of multielectron $(N \geqslant 2)$ atomic [14-16] and molecular $[16,17]$ systems in intense laser fields, where the dynamical electron-correlation is treated by approximate exchangecorrelation energy functionals. However, exact treatment of electron correlation of many-electron quantum systems in time-dependent fields remains to be developed.

In this paper, we present a fully ab initio wave function approach for nonperturbative treatment of two-electron atomic systems in intense laser fields. The method is based on the extension of the time-dependent generalized pseudospectral (TDGPS) method [18] to the three-body quantum systems in hyperspherical coordinates (HSC) $[19,20]$. In the TDGPS approach, the GPS technique [21] is used for nonuniform and optimal discretization of the spatial coordinates and a second-order split-operator method in the energy representation is used for efficient and accurate time propagation of the wave function. The TDGPS method has been shown to be capable of providing highly accurate timedependent wave functions for both HHG and MPI calculations $[14,16-18]$ and high-resolution spectroscopy of fieldinduced Rydberg atoms [22], with the use of only a modest number of spatial grid points. Moreover, the use of the energy representation facilitates the elimination of undesirable high-energy (fast oscillating) components, allowing the use of larger time step and more efficient time propagation of the wave function. For the two-electron atomic systems, the study of the electron correlation effects can be facilitated by the use of the hyperspherical coordinate formalism. In this paper, we present a TDGPS-HSC method for accurate nonperturbative treatment of the quantum dynamics and the role of electron correlation on the HHG processes of $\mathrm{He}$ atoms in intense ultrashort laser pulses. A related approach has been recently presented for the study of double photoexcitation of 
He atoms by attosecond XUV pulses in the presence of weak few-cycle infrared lasers [23].

The paper is organized as follows. In Sec. II, we present the detailed TDGPS-HSC procedure for nonperturbative treatment of HHG processes of two-electron atoms in intense laser pulses. Section III presents the calculations of the doubly excited states, oscillator strengths, radial probability distribution, and HHG power spectra of He atoms. Exploration of the effects of electron correlation and doubly excited states on HHG are discussed in detail. Conclusions and remarks are presented in Sec. IV. Atomic units are used through the present work unless otherwise indicated.

\section{BASIC FORMULATION}

\section{A. Hyperspherical coordinate formalism and the time- dependent generalized pseudospectral method for spatial discretization and time propagation}

In a recent work, Tong and Lin [23] have explored the influence of weak laser pulses $\left(I_{0} \leqslant 10^{12} \mathrm{~W} / \mathrm{cm}^{2}\right)$ on the double photonabsorption cross sections of helium by using the HSC approach (combined with the $R$-matrix theory). In the present work, the hyperspherical couple-channel approach is extended to the nonperturbative treatment of the response of the helium atoms to intense laser pulse $\left(I_{0} \geqslant 1\right.$ $\times 10^{14} \mathrm{~W} / \mathrm{cm}^{2}$ ) fields. The pulse duration considered ranges from 5 to $25 \mathrm{fs}$. One of the advantages of the HSC approach is that the two correlated electrons can be treated on equal footing and no single-electron orbitals are involved to construct wave functions of interest. This allows us to be able to consider those dynamical processes in which both electrons are active.

In this work, the wave function in laser pulses is expanded in terms of the field-free eigenchannels (see below) and the time-dependent coefficients can be obtained by propagating the wave function (initially from the He ground state) by means of the TDGPS procedure $[18,22]$. In the following, we summarize some essential points of the hyperspherical couple-channel formalism and present the extension of the TDGPS technique for the optimal spatial discretization and time propagation of the wave function in hyperspherical coordinates.

In the HSC formalism, the Hamiltonian of the helium atom in field-free case can be written as

$$
H \Phi\left(R, \alpha, \Omega_{1}, \Omega_{2}\right)=E \Phi\left(R, \alpha, \Omega_{1}, \Omega_{2}\right)
$$

where the hyperradius, $R=\sqrt{r_{1}^{2}+r_{2}^{2}}$, is a measure of the spatial size, and the hyperangle, $\alpha=\tan ^{-1}\left(r_{2} / r_{1}\right)$, depicts the radial correlation, are introduced. Here $\Omega_{i}(i=1$ and 2) stands for the spherical angles $\theta_{i}$ and $\varphi_{i}$, respectively. Note that the so-called angular correlation is described by the angle $\theta_{12}$ between the two radial vectors. In Eq. (1), we have

$$
H=-\frac{1}{2}\left[\frac{\partial^{2}}{\partial^{2} R}+\frac{5}{R} \frac{\partial}{\partial R}\right]+\frac{\Lambda^{2}}{2 R^{2}}+\frac{C}{R},
$$

$$
\begin{aligned}
\Lambda^{2}= & -\frac{1}{\sin ^{2} \alpha \cos ^{2} \alpha} \frac{\partial}{\partial \alpha}\left(\sin ^{2} \alpha \cos ^{2} \alpha \frac{\partial}{\partial \alpha}\right) \\
& +\frac{\hat{\ell}_{1}^{2}}{\cos ^{2} \alpha}+\frac{\hat{\ell}_{2}^{2}}{\sin ^{2} \alpha},
\end{aligned}
$$

and

$$
C\left(\alpha, \theta_{12}\right)=-\frac{2}{\sin \alpha}-\frac{2}{\cos \alpha}+\frac{1}{\sqrt{1-\sin (2 \alpha) \cos \left(\theta_{12}\right)}},
$$

where $C / R$ includes all the Coulomb interactions within the atomic system. To eliminate the first derivative with respect to the hyperradius $R$ in Eq. (2), let

$$
\Phi\left(R, \alpha, \Omega_{1}, \Omega_{2}\right)=\frac{\Psi\left(R, \alpha, \Omega_{1}, \Omega_{2}\right)}{R^{5 / 2} \sin \alpha \cos \alpha} .
$$

We thus have the Schrödinger equation in the reduced space

$$
\begin{aligned}
& {\left[-\frac{1}{2} \frac{\partial^{2}}{\partial^{2} R}-\frac{1}{8 R^{2}}+\frac{1}{2 R^{2}}\left(-\frac{\partial^{2}}{\partial^{2} \alpha}+\frac{\hat{\ell}_{1}^{2}}{\cos ^{2} \alpha}+\frac{\hat{\ell}_{2}^{2}}{\sin ^{2} \alpha}\right)+\frac{C}{R}\right] \Psi} \\
& \quad=E \Psi .
\end{aligned}
$$

With the above definitions, it is convenient to introduce the adiabatic eigenchannel representation to facilitate the solution of the above-mentioned coupled channel equations. Once we have obtained the eigenchannel functions which are the essential ingredient in the HSC approach and the relevant adiabatic potential curves, we can use them to construct the whole solution to Eq. (6). For a fixed $R$, we have

$$
\begin{aligned}
& {\left[\frac{1}{2 R^{2}}\left(-\frac{\partial^{2}}{\partial \alpha^{2}}+\frac{\hat{\ell}_{1}^{2}}{\cos ^{2} \alpha}+\frac{\hat{\ell}_{2}^{2}}{\sin ^{2} \alpha}\right)+\frac{C}{R}\right] \Phi_{\mu}\left(R, \alpha, \Omega_{1}, \Omega_{2}\right)} \\
& \quad=U_{\mu}(R) \Phi_{\mu}\left(R, \alpha, \Omega_{1}, \Omega_{2}\right) .
\end{aligned}
$$

In the following we discuss the procedure for achieving accurate solution to the above equation. A well-known method is the Numerov numerical integration of the coupled equations [19]. Another one is the basis set expansion [20] in term of eigenfunctions of hyperspherical harmonics. However, it is not so hard to recognize that the latter suffers from the difficulty of slow convergence. This is especially true in the region far from the nucleus, because one needs to use considerably more coupled hyperspherical harmonics to describe the hydrogenlike functions. In other words, this method provides an inadequate description at larger $R$ and thus it is not appropriate for our present goal to describe the laser-driven helium atom. In this work we develop an effective GPS techinque in hyperspherical coordinates for nonuniform and optimal spatial discretization and solution of adiabatic coupled equations, i.e., a strategy to discretize both hyperradius and hyperangle variables to overpass the abovementioned obstacles. Let us introduce an auxiliary variable $y=4 \alpha / \pi-1$ to map the hyperangle $\alpha$ into the domain $y \in[-1,+1]$. For a given hyperradius, the discretized twoelectron wave function within channel $\mu$ is expressed as 


$$
\begin{aligned}
\Phi_{\mu}\left(x, y, \Omega_{1}, \Omega_{2}\right)= & \sum_{\left\{l_{1}, l_{2}\right\}}\left[\sum_{k}^{N_{\alpha}} f_{k}(y) F_{l_{1} l_{2} \mu}^{L M}\left(x, y_{k}\right) \mathcal{Y}_{l_{1} l_{2}}^{L M}\left(\Omega_{1}, \Omega_{2}\right)\right. \\
& +(-1)^{\delta} \sum_{k}^{N_{\alpha}} f_{k}(-y) F_{l_{1} l_{2} \mu}^{L M}\left(x, y_{k}\right) \mathcal{Y}_{l_{2} l_{1}}^{L M} \\
& \left.\times\left(\Omega_{1}, \Omega_{2}\right)\right],
\end{aligned}
$$

where we define $\delta=l_{1}+l_{2}-L+S$. $L$ and $S$ indicate the total orbital angular and spin momentum, respectively.

Because the total angular and spin momenta are explicitly involved in the wave function defined in Eq. (8), here we see that the above discretized channel function satisfies all the requirement of exchange symmetries, i.e., its spatial part is exchange symmetric for the triplet states while antisymmetric for the singlet states. The first advantage in the present GPS procedure is in that we can completely separate the singlet states from the triplet ones. At the first glance, a simplified version of the discretized representation by dropping the second summation term in Eq. (8) may be working well. However, it does not meet our present purpose to handle the two-electron response to laser pulses as explained below. Dropping the term mentioned above results in the mixture of the singlet and triplet states and one has to filter out the potential curves of the singlet states from the triplet ones. For the laser intensity of $10^{14}-10^{15} \mathrm{~W} / \mathrm{cm}^{2}$ of the present interest, the relativistic effect caused by intense laser pulses can be safely neglected. That means no coupling between the singlet and triplet states exist and therefore the total spin angular momentum must be a conserved quantity. The second advantage is that the boundary conditions imposed in the channel functions, $\Psi(R, \alpha=0)=0$ and $\Psi(R, \alpha=\pi / 2)=0$, are explicitly taken into account through the cardinal function $f(y)$ defined by the Gauss-Lobatto quadrature. Another advantage of the present GPS technique is that it does not require to calculate the complicated and time-consuming matrix elements of the Coulomb repulsion interaction, since only the values of the Coulomb potentials in the spatial mesh are needed. Furthermore both the hyperradius $R$ and hyperangle $\alpha$ are discreized in an optimal and nonuniform fashion, namely, more grid points are placed nearby the nucleus and sparser grid structure outward, with the use of only a modest number of grid points. This speeds up considerably the numerical calculation and at the same time provides accurate wave function at the grid points.

As an example of the accuracy of the present GPS procedure, Fig. 1 shows the first ten adiabatic potential curves of helium in the singlet ${ }^{1} S^{e},{ }^{1} P^{o}$, and ${ }^{1} D^{e}(L=0-2)$ manifolds converging to the $\mathrm{He}^{+}$ionization thresholds. We note that there is a number of avoided crossings even between the low-lying neighboring potential curves. For example, a sharply avoided crossing point in the ${ }^{1} P_{o}$ manifold occurs around the hyperradius of $7.65 \mathrm{a} . \mathrm{u}$.

Next let us discuss on how to solve the Schrödinger equation in the hyperradius space, once the information of eigenchannels is available. Similar to the situation in a molecular system, we can think of the hyperradius $R$ as an adiabatic or

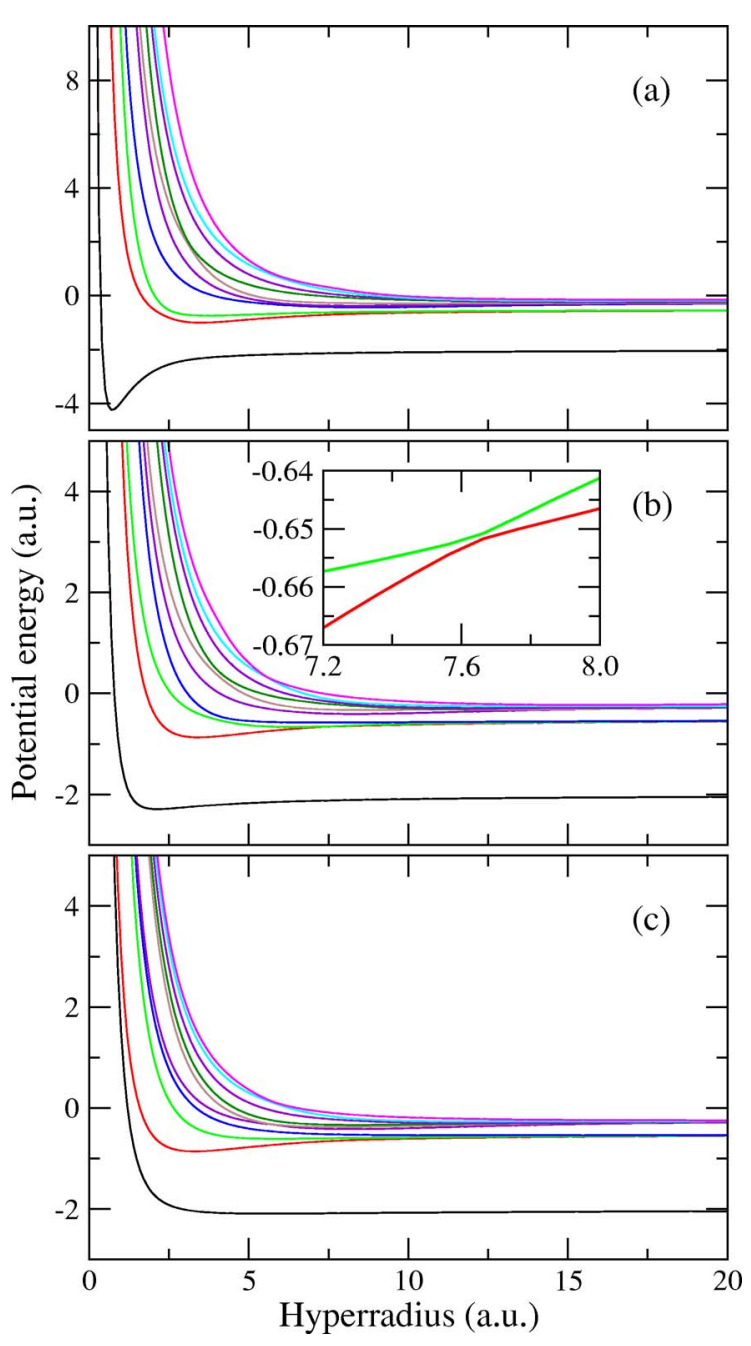

FIG. 1. (Color online) Potential curves of the helium atom for (a) ${ }^{1} S^{e}$, (b) ${ }^{1} P^{o}$, and (c) ${ }^{1} D^{e}$ manifolds.

"smooth" variable [24]. Most of the kinetic energy of the two-electron atom is contributed from the motion related to the hyperangle degree of freedom, while the hyperradius motion contributes a minor portion. With this in mind and after mapping the hyperradius $R$ in a finite box $\left[0, R_{\max }\right]$ into the domain $[-1,+1]$ by

$$
R(x)=R_{0} \frac{1+x}{1-x+\gamma},
$$

where $\gamma=2 R_{0} / R_{\text {max }}$, we obtain the following transformation:

$$
\begin{aligned}
\Psi\left(R, \alpha, \Omega_{1}, \Omega_{2}\right) & =\sqrt{R^{\prime}(x)} \sum_{i=1}^{N_{r}} f_{i}(x) \Psi\left(x_{i}, y, \Omega_{1}, \Omega_{2}\right) \\
& =\sqrt{R^{\prime}(x)} \sum_{i \mu}^{N_{r} N_{\mu}} f_{i}(x) C_{\mu i} \Phi_{\mu}\left(x_{i}, y, \Omega_{1}, \Omega_{2}\right) .
\end{aligned}
$$

Inserting the discretized representation of wave function into Eq. (6), we can rewrite the discretized Schrödinger equation in the form 


$$
\begin{gathered}
\sum_{i^{\prime} \mu^{\prime}}\left[\mathcal{K}_{i i^{\prime}} \mathcal{O}_{i \mu, i^{\prime} \mu^{\prime}}+\delta_{i i^{\prime}} U_{\mu^{\prime}}\left(R_{i^{\prime}}\right) \mathcal{O}_{i \mu, i^{\prime} \mu^{\prime}}\right] C_{\mu^{\prime} i^{\prime}} \\
=E \sum_{i^{\prime} \mu^{\prime}} \delta_{i i^{\prime}} \mathcal{O}_{i \mu, i^{\prime} \mu^{\prime}} C_{\mu^{\prime} i^{\prime}}
\end{gathered}
$$

where the matrix element of kinetic energy, $\mathcal{K}_{i i^{\prime}}$, in the hyperradius direction is defined by

$$
\mathcal{K}_{i i^{\prime}}=-\left.\frac{1}{2}\left(\frac{\partial^{2}}{\partial R^{2}}-\frac{15}{4 R^{2}}\right) f_{i^{\prime}}(R)\right|_{R=R_{i}} .
$$

Note that the electron-electron interaction has already been incorporated in the adiabatic potential energy $U_{\mu}(R)$. One of the appealing features in the present scheme is that one does not need to calculate explicitly non-adiabatic coupling between eigenchannels. In the Ref. [24], the method to discretize the hyperradius is referred as smooth variable discretization (SVD). Our procedure differs from the SVD in that a nonlinear transformation [see Eq. (9)] of the hyperradius $R$ is introduced for accurate treatment of the Coulomb singularity nearby the nucleus.

Because the two active electrons are confined in a finite box [see Eq. (9) for $R_{\text {max }}$ ], all the eigenenergies turned out to be discretized. This indicates that the bound and continuum states can be taken into account in the same way. It provides an accessible way to control the density of states in the continuum by adjusting the parameters involved in nonlinear mapping or the number of grid points. This allows us to be able to consider a variety of the ionization processes induced by intense laser pulses.

\section{B. TDGPS method for time-propagation of the laser-driven helium-atom wave function in discretized hyperspherical coordinates system}

Application of hyperspherical coordinates technique to the few-electron atomic systems in the field-free case is far from new $[19,20]$, however, its time-dependent version is still under development. Numerical stable and accurate algorithms are required for the treatment of the interaction of the atomic system with intense laser fields and the physical quantities of interest can be then extracted from the timedependent wave functions. In the present problem, we generalize the TDGPS techniques [18,22] to the hyperspherical coordinates for nonuniform spatial discretization of the coupled-channel Hamiltonian and for performing the time propagation of the wave function by means of the secondorder split-operator technique in the energy representation. If we choose the adiabatic eigenchannel functions as our channel basis functions to evolve the time-dependent wave function, namely

$$
\Psi\left(R, \alpha, \Omega_{1}, \Omega_{2}, t\right)=\sum_{L \mu} F_{\mu}^{L}(R, t) \Phi_{\mu}^{L}\left(R, \alpha, \Omega_{1}, \Omega_{2}\right),
$$

then the coupled equations take the form

$$
\begin{aligned}
\mathrm{i} \sum_{L^{\prime} \mu^{\prime} i^{\prime}} \frac{\partial F_{\mu^{\prime}}^{L^{\prime}}\left(R_{i^{\prime}}, t\right)}{\partial t} \mathcal{O}_{L \mu i}^{L^{\prime} \mu^{\prime} i^{\prime}}= & \sum_{L^{\prime} \mu^{\prime} i^{\prime}} F_{\mu^{\prime}}^{L^{\prime}}\left(R_{i^{\prime}}, t\right)\left[\mathcal{K}_{i^{\prime} i} \mathcal{O}_{L \mu i}^{L^{\prime} \mu^{\prime} i^{\prime}}\right. \\
& \left.+\mathcal{H}_{i i^{\prime}}^{a d}+\mathcal{V}_{i i^{\prime}}(t)\right]
\end{aligned}
$$

Equation (14) is solved by the second-order split-operator technique in the energy representation to propagate the twoelectron wave packet, which is initially launched from its strongly correlated ground state. We begin by writing the evolution equation

$$
\Psi(R, t+\Delta t)=e^{-i H_{0} \Delta t / 2} e^{-i V(t+\Delta t / 2) \Delta t} e^{-i H_{0} \Delta t / 2} \Psi(R, t)+O(\Delta t)^{2},
$$

where $H_{0}$ stands for the field-free Hamiltonian and $V(t)$ is the interaction of the helium atom with laser pulses in the length gauge. Here we assume that the electric field $\boldsymbol{E}(t)$ has a $\sin ^{2}$ envelope function $f(t)$ and is linearly polarized along the $z$ axis. In the energy representation [18], it is essential to obtain the so called $S$ matrix in hyperspherical space, which indicates the effect of the exponential operator $e^{-i H_{0} \Delta t / 2}$ on the $\Psi(R, t)$ in Eq. (15). Here the $S$-matrix element reads

$$
\mathcal{S}_{i i^{\prime}}^{\mu \mu^{\prime} L}(\Delta t)=\omega_{i} R^{\prime 2}\left(x_{i}\right) \sum_{\mu^{\prime \prime}} e^{-i \varepsilon_{\mu^{\prime \prime}}^{L} \Delta t / 2} C_{i \mu}^{L \mu^{\prime \prime}} C_{i^{\prime} \mu^{\prime}}^{L \mu^{\prime \prime}},
$$

where $\varepsilon_{\mu^{\prime \prime}}^{L}$ denotes the energy spectra of helium in the fieldfree case. Therefore, the channel component $F_{\mu}^{L}\left(R_{i}, t\right)$ on the mesh can be calculated through the following three successive time steps

$$
\begin{gathered}
F_{\mu}^{L(1)}\left(R_{i}, t+\Delta t\right)=\sum_{i^{\prime} \mu^{\prime}} F_{\mu^{\prime}}^{L(0)}\left(R_{i^{\prime}}, t\right) \mathcal{S}_{i i^{\prime}}^{\mu \mu^{\prime} L}(\Delta t) ; \\
F_{\mu}^{L(2)}\left(R_{i}, t+\Delta t\right)=\sum_{L^{\prime} \mu^{\prime}} F_{\mu^{\prime}}^{L^{\prime}(1)}\left(R_{i}, t+\Delta t\right) \mathcal{Z}_{L \mu}^{L^{\prime} \mu^{\prime}}(\Delta t) ; \\
F_{\mu}^{L(3)}\left(R_{i}, t+\Delta t\right)=\sum_{i^{\prime} \mu^{\prime}} F_{\mu^{\prime}}^{L(2)}\left(R_{i^{\prime}}, t+\Delta t\right) \mathcal{S}_{i i^{\prime}}^{\mu \mu^{\prime} L}(\Delta t) .
\end{gathered}
$$

Based on the equations above, we can recast the timeevolution of the wave function into the following matrix form

$$
\boldsymbol{F}(t+\Delta t)=\boldsymbol{S Z}(t+\Delta t) \boldsymbol{S} \boldsymbol{F}(t)=\boldsymbol{M}(t+\Delta t) \boldsymbol{F}(t) .
$$

The above scheme of time evolution is quite general in the sense that it can be applied either for the cases of timedependent fields or for the cases of time-independent ones. For our present case, the $\boldsymbol{Z}$ matrix caused by the external laser fields is explicitly dependent on time, while the $S$ matrix is independent of time. This means that the $S$ matrix needs to be calculated only once, before the time-evolution starts. We note that all the evolution matrices are required to be determined only once if the external fields are timeindependent (see, for example, for atoms in static magnetic and/or crossed electric and magnetic fields) [22]. 
Once the time-dependent wave functions are calculated, the induced dipole length and acceleration by laser pulses can be determined by

$$
d_{L}(t)=\left\langle\Psi(t)\left|\sum_{i=1}^{2} z_{i}\right| \Psi(t)\right\rangle,
$$

and

$$
d_{A}(t)=\left\langle\Psi(t)\left|\sum_{i=1}^{2}\left[-\frac{z_{i}}{r_{i}^{3}}+E_{0} f(t) \sin \left(\omega_{0} t\right)\right]\right| \Psi(t)\right\rangle,
$$

respectively. From a classical point of view, for a multielectron system, the electron-electron Coulomb interaction does not explicitly contribute to the acceleration as we can see from Eq. (20). In the acceleration gauge, if we take the power spectrum to have the form

$$
P_{A}(\omega)=\left|\frac{1}{\omega^{2} \tau} \int_{0}^{\tau} d_{A}(t) e^{i \omega t} \mathrm{~d} t\right|^{2},
$$

then we have an equivalent form in the length gauge

$$
P_{L}(\omega)=\left|\frac{1}{\tau} \int_{0}^{\tau} d_{L}(t) e^{i \omega t} \mathrm{~d} t-\frac{\dot{d}_{L}(\tau)}{\omega^{2} \tau} e^{i \omega \tau}+\mathrm{i} \frac{d_{L}(\tau)}{\omega \tau} e^{i \omega \tau}\right|^{2} .
$$

Here $\dot{d}_{L}(\tau)$ stands for the first derivative of $d_{L}(t)$ at the end of laser pulses. Note that the first integral in Eq. (22) alone has been widely employed in the literature to calculate the HHG power spectrum in the dipole length form and the last two terms are often dropped. However, this is valid only when both $\dot{d}_{L}(\tau)$ and $d_{L}(\tau)$ vanish or are negligibly small [25].

\section{RESULTS AND DISCUSSIONS}

\section{A. The helium atom in the field-free case}

Since the time-dependent wave functions of interest are expanded in terms of the field-free adiabatic eigenchannels, it is instructive to briefly recap some of results on the helium atom in the field-free case, before we turn to discuss the response to time-dependent laser fields. To illustrate the accuracy of the present methods, we list the energies of singly as well as doubly excited states in Table I, while selected oscillator strengths between low-lying states are listed in Table II. When we incorporated ten eigenchannels and 11 angular components in the wave function, the ground state energy of helium is determined to be -2.903723 a.u., in excellent agreement with the benchmark result of -2.903724 a.u. [26]. In the present calculations, we have used $N_{R}=100$ and $N_{\alpha}=150$ grid points. Such an accuracy reported here cannot be achieved by means of commonly used equal-spacing discretization methods without the use of many-orders-of-magnitude larger number of grid points. The accuracy of the field-free energies listed is better than $10^{-5}$ a.u. In Table II, the field-free oscillator strengths of the transitions 1 snp ${ }^{1} P \rightarrow 1 s^{2}{ }^{1} S$ are extracted from the Fourier
TABLE I. Energies of the singly and doubly excited states of the helium atom. No effect of finite nuclear mass is included in the present and literature values. Underlined digits indicate uncertainties in Ref. [27]. All energies are given in a.u.

\begin{tabular}{lcc}
\hline \hline & Singly excited states \\
\hline & This work & Ref. [26] \\
$1 s^{21} S^{e}$ & -2.903723 & -2.903724 \\
$1 s 2 s^{1} S^{e}$ & -2.145969 & -2.145974 \\
$1 s 2 p^{1} P^{e}$ & -2.123824 & -2.123843 \\
$1 s 3 s^{1} S^{e}$ & -2.061261 & -2.061271 \\
$1 s 3 p^{1} P^{e}$ & -2.055127 & -2.055146 \\
$1 s 3 d^{1} D^{e}$ & -2.055600 & -2.055620
\end{tabular}

Doubly excited states

below the $\mathrm{He}^{+} n=2$ threshold

This work

Ref. [27]

${ }_{2}(1,0)_{2}^{+1} S^{e}$

$-0.77781$

$-0.77787$

${ }_{2}(-1,0)_{2}^{+1} S^{e}$

$-0.62195$

$-0.621 \underline{81}$

${ }_{2}(-1,0)_{3}^{+1} S^{e}$

$-0.54805$

$-0.548070$

${ }_{2}(0,1)_{2}^{+1} P^{o}$

$-0.69321$

$-0.693069$

${ }_{2}(1,0)_{3}^{-1} P^{o}$

$-0.59704$

$-0.597074$

${ }_{2}(0,1)_{3}^{+1} P^{o}$

$-0.56404$

$-0.564074$

${ }_{2}(-1,0)_{3}^{01} P^{o}$

$-0.54707$

$-0.547087$

transform of the autocorrelation functions [22,28]. Meanwhile, time-dependent wave functions are obtained by propagating the initial wave packet within a TDGPS scheme outlined in the last section. The results obtained by the timedependent and time-independent methods are in good agreement with each other and also with the exact reference values [26], indicating sufficient accuracy has been achieved by the present calculations.

\section{B. Single-atom nonlinear harmonic response}

For the wavelength of $248.6 \mathrm{~nm}$ used in this study, only six photons are required to produce the single ionized $\mathrm{He}^{+}$ ion. The ponderomotive energy of the laser-driven free electron at a laser intensity of $5 \times 10^{14} \mathrm{~W} / \mathrm{cm}^{2}$ is 0.105 a.u.,

TABLE II. Comparison of the oscillator strengths of the helium atom. The methods (a) and (b) indicate the present timeindependent and time-dependent calculations, respectively (see the text). The initial state is chosen as the ground state of helium and no effect of finite nuclear mass is included. All energies are in a.u.

\begin{tabular}{lcccc}
\hline \hline$n$ & Energy & Method (a) & Method (b) & Ref. [26] \\
\hline 2 & -2.123824 & 0.276167 & 0.276166 & 0.2761647 \\
3 & -2.055127 & 0.073416 & 0.073415 & 0.0734349 \\
4 & -2.031057 & 0.029851 & 0.029850 & 0.0298629 \\
5 & -2.019898 & 0.015034 & 0.015034 & 0.0150393 \\
6 & -2.013811 & 0.008614 & 0.008613 & 0.0086277 \\
\hline \hline
\end{tabular}


while the quiver amplitude is smaller than 3.55 a.u. Compared with the cases of atoms at longer wavelengths such as of 780 or $1064 \mathrm{~nm}$, the ponderomotive energy gained by the ionizing electron in one optical period is considerably smaller. As a consequence, this leads to the absence of the "shoulder" structure in cross section of the double ionization. This means that the whole ionization process is dominated by the sequential events, rather than by the nonsequential ones in the present case. This point has been demonstrated experimentally in Ref. [29].

Depending on the laser parameters, the hyperspherical space is discretized on a mesh of $N_{R} \times N_{\alpha}=200 \times 80$. In general, only three eigenchannels and six eigenmanifolds (i.e., $0 \leqslant L \leqslant 5$ ) are sufficient to achieve the converged results. The size of the problem may be effectively reduced if the zerofield eigenmanifolds are properly chosen. For example, the manifold of $[(1,1) 1]^{1} P^{e}$ is excluded from the expansions of wave functions due to the selection rule. In order to gain more insight into the response of helium to laser pulses, the two-electron joint radial probability distribution is illustrated in Fig. 2 for the case of $I_{0}=1 \times 10^{14} \mathrm{~W} / \mathrm{cm}^{2}$ and $\tau=6$ optical cycles (o.c.) The radial probability density is defined by integrating all the angle variables

$$
\begin{aligned}
P\left(r_{1}, r_{2}, t\right)= & r_{1}^{2} r_{2}^{2} \iint \Psi^{*}\left(R, \alpha, \Omega_{1}, \Omega_{2}, t\right) \Psi\left(R, \alpha, \Omega_{1}, \Omega_{2}, t\right) \\
& \times \mathrm{d} \Omega_{1} \mathrm{~d} \Omega_{2} .
\end{aligned}
$$

We note that the electronic density is distributed in the region where only one electron is driven to travel over the region far from nucleus while another one is still confined near the nucleus. As expected, a clear indication of the single ionization process can be seen.

Since we are working in hyperspherical coordinate framework, it is instructive to observe the time evolution of the spatial size of helium through the well-defined hyperradius. But note that the so-called time-dependent hyperangle $\alpha(t)$ defined by $\alpha(t)=\langle\Psi(t)|\alpha| \Psi(t)\rangle$ does not provide any information on the dynamics, because we have always $\alpha(t)$ $\equiv \pi / 4$. From Fig. 3 , for the case of $\tau=30$ o.c., the hyperradius goes through the global maximum at $t=15$ o.c., when the laser pulse rises to its peak intensity. By comparing the behavior of the time-dependent hyperradius $\langle R(t)\rangle$ and the single-electron radius $\langle r(t)\rangle\left(=\left\langle r_{1}(t)+r_{2}(t)\right\rangle / 2\right)$, it is realized that for the laser parameters used here the single-electron dynamics dominates others. This indicates that one electron is bound in the ground state of the parent ion while another one is ionized. Therefore, $\langle R(t)\rangle$ and $\langle r(t)\rangle$ show a similar response to laser pulse. Also, the spatial size of the laserdriven helium atom shows a periodical oscillation with the period of half optical cycle of laser electric fields. On the other hand, as revealed in Fig. 3, the spatial extension does not grow so much during the time evolution, because of the smaller quiver amplitude. From a semiclassical viewpoint $[30,31]$, the time-dependent laser electric field changes its direction with time, the ionizing electron is thus driven back periodically and then to recollide with its parent $\mathrm{He}^{+}$ion.
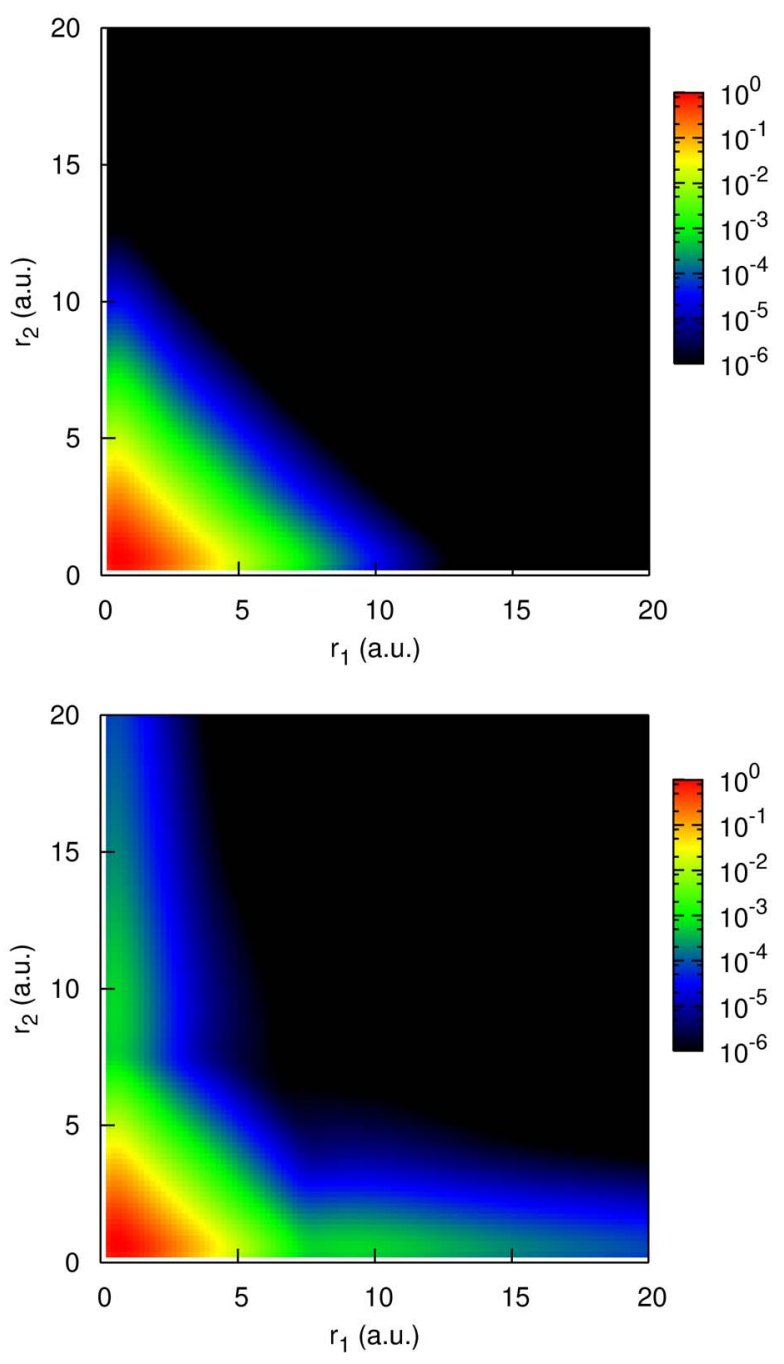

FIG. 2. (Color online) Snapshots of the two-electron helium radial density in the $r_{1}-r_{2}$ plane in a $248.6 \mathrm{~nm}$ laser pulse with intensity $1 \times 10^{14} \mathrm{~W} / \mathrm{cm}^{2}$, taken at (a) $t=0$ and (b) $t=\tau / 2$. The time duration is $\tau=6$ o.c. The colors are shown in logarithmic scale (in the powers of 10).

As an example, Fig. 4 shows the induced dipole length and acceleration in a $\sin ^{2}$ laser pulse. The corresponding HHG power spectra are displayed in Fig. 5 for comparison. As shown, the present ab initio HHG power spectra in the dipole and acceleration forms are in very good agreement with each other even in the region beyond the cutoff. This indicates that the convergence of the time-dependent wave functions has been achieved in the whole configuration space, both in the region near the atomic core as well as in the region far from nucleus.

Figures 6 and 7 show the comparison of the HHG power spectrum of the fully two-electron and the SAE calculations in two different laser intensities. First we notice that there exists some unique broader harmonic peaks with spectral line profile seen only in the fully $a b$ initio results. Figure 7 shows that the $a b$ initio HHG power spectrum contains a broad peak with fine structure at the fifth harmonic at the peak intensity of $I_{0}=5 \times 10^{14} \mathrm{~W} / \mathrm{cm}^{2}$. This broader structure can be attributed to the resonance between the intermediate 


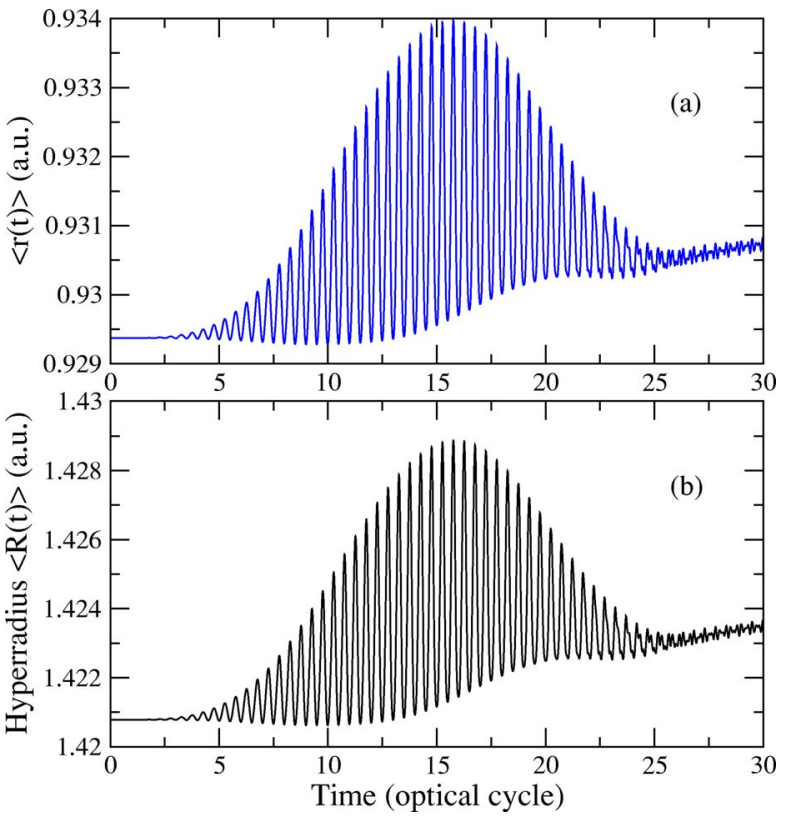

FIG. 3. (Color online) Time evolution of (a) the $\left\langle r_{1}+r_{2}\right\rangle / 2$ and (b) the hyperradius $\langle R(t)\rangle$ of the helium atom in the $\sin ^{2}$ laser pulses. The laser parameters are peak intensity $I_{0}=1$ $\times 10^{14} \mathrm{~W} / \mathrm{cm}^{2}$, wavelength $\lambda=248.6 \mathrm{~nm}$ and time duration $30 \mathrm{op}-$ tical cycles.

$1 s 2 p^{1} P^{o}$ state and the ground state in intense laser pulses. Note that at the same wavelength $(248.6 \mathrm{~nm})$, Glass and Burke [32] found that the five-photon resonance between the intermediate $1 s 2 p$ state and the ground state occurs just at $I_{0}=5 \times 10^{14} \mathrm{~W} / \mathrm{cm}^{2}$. In Ref. [32], this five-photon resonance was explored by means of the total ionization rate and the

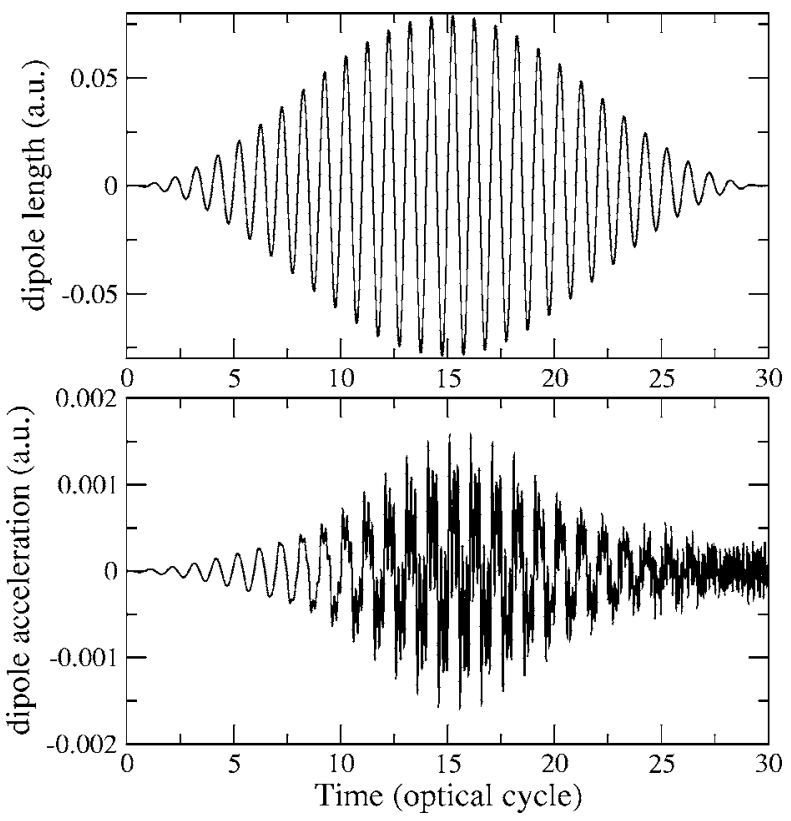

FIG. 4. Time-dependent dipole length and acceleration of the helium atom in the $\sin ^{2}$ laser pulses with peak intensity of 1 $\times 10^{14} \mathrm{~W} / \mathrm{cm}^{2}$. The laser pulse has a wavelength of $248.6 \mathrm{~nm}$ and a time duration of 30 optical cycles.

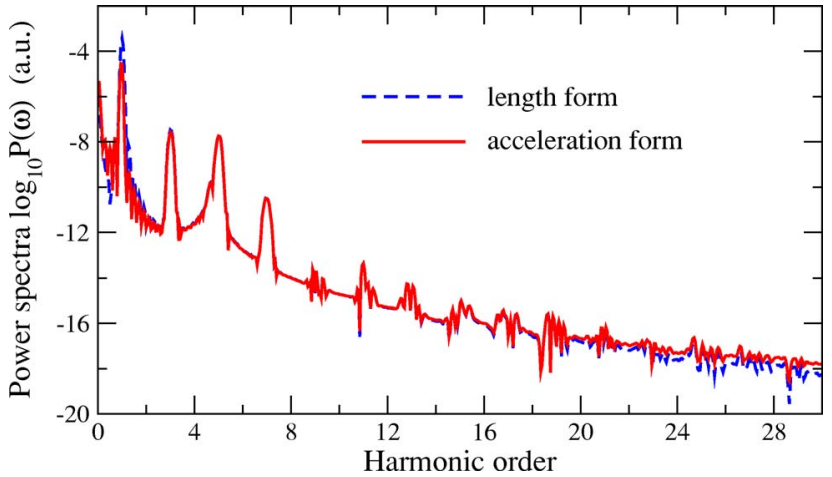

FIG. 5. (Color online) Harmonic generation of the helium atom in the $\sin ^{2}$ laser pulses with peak intensity of $I_{0}=1 \times 10^{14} \mathrm{~W} / \mathrm{cm}^{2}$. The laser pulse has a wavelength of $248.6 \mathrm{~nm}$ and a time duration of 15 optical cycles.

energies of Rydberg states. However, at a weaker intensity of $1 \times 10^{14} \mathrm{~W} / \mathrm{cm}^{2}$ (see Fig. 6), a broader resonance with a Fano line shape occurs at a higher energy regime where the energy of the emitted photon is $13 \omega_{0}$. This energy scale is far above the first ionization threshold. To gain a deeper insight into the coupling between the doubly excited states and ground state caused by the laser pulses, Fig. 8 displays the emitted spectra by including only the first eigenchannel converging to the $\mathrm{He}^{+} n=1$ threshold (dashed line). In this way, the coupling between the doubly excited states and ground state are removed and only the effect of the single excited Rydberg states are taken into account. As a consequence, we note that the broader resonance structure completely disappeared in the energy regime near the 13th harmonic, the latter now shows a similar resonance width to the neighboring harmonics. From Table I, we further notice that the broader resonance at the frequency of $13 \omega_{0}$ can be attributed to the multiphoton resonance between a group of the doubly excited ${ }_{2}(1,0)_{3}^{-1} P^{o},{ }_{2}(0,1)_{3}^{+1} P^{o}$, and ${ }_{2}(-1,0)_{3}^{01} P^{0}$ states and the ground state. For the laser parameters in Fig. 8, we have the energy difference $\Delta E \simeq E_{d e}^{\prime}\left({ }^{1} P^{0}\right)-E_{g}^{\prime}\left({ }^{1} S^{e}\right)=2.36$ a. u. $\simeq 13 \omega_{0}$.

It is instructive to explore whether a SAE model can be used to describe the process in which the sequential event

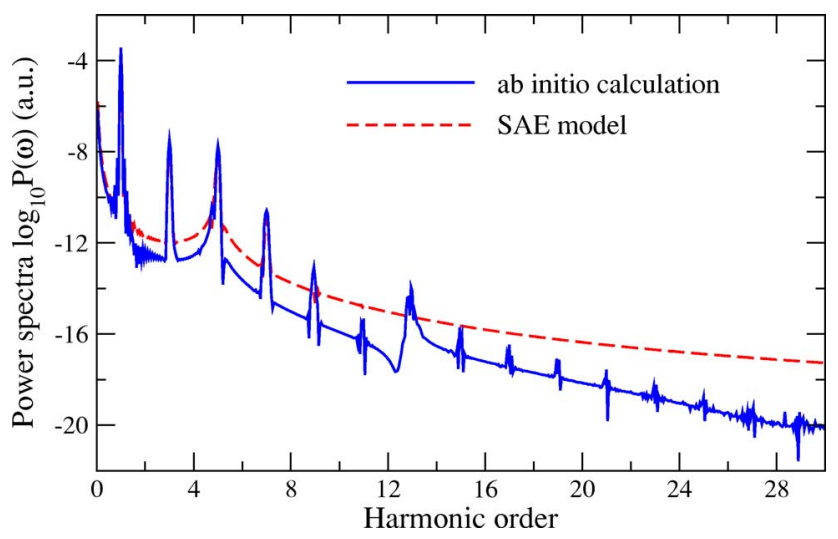

FIG. 6. (Color online) Comparison of HHG power spectra obtained by the SAE model and the full-dimensional helium theory. The laser pulse has a wavelength of $248.6 \mathrm{~nm}$, a peak intensity of $I_{0}=1 \times 10^{14} \mathrm{~W} / \mathrm{cm}^{2}$ and a time duration $\tau=30$ optical cycles. 


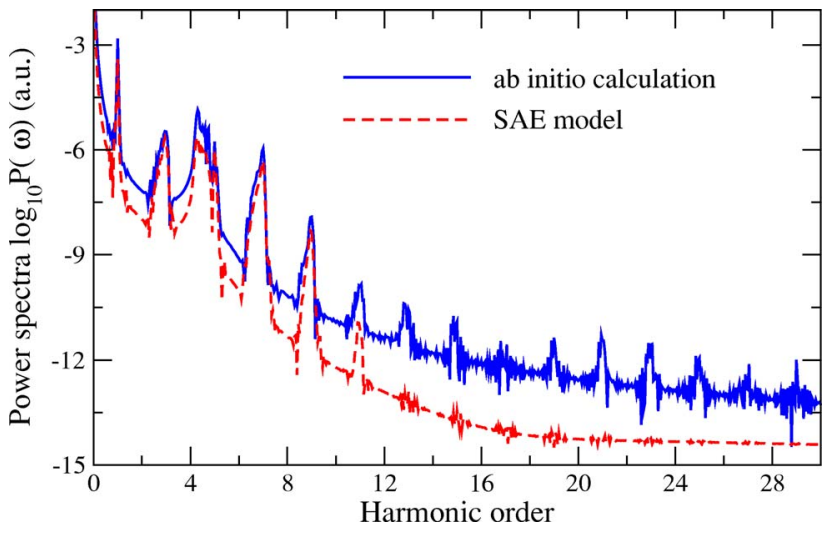

FIG. 7. (Color online) Same as Fig. 6, but for the laser parameters of peak intensity $I_{0}=5 \times 10^{14} \mathrm{~W} / \mathrm{cm}^{2}$ and time duration $\tau$ $=30$ optical cycles.

dominates others. In Figs. 6 and 7 we compare the power spectra obtained by the ab initio two-electron hyperspherical approach and the SAE model. From Fig. 6, we see the SAE plateau is extended only to the about the fifth order in the emitted spectra. This is in accordance with the prediction of the semiclassical recollision model [30,31], which neglects

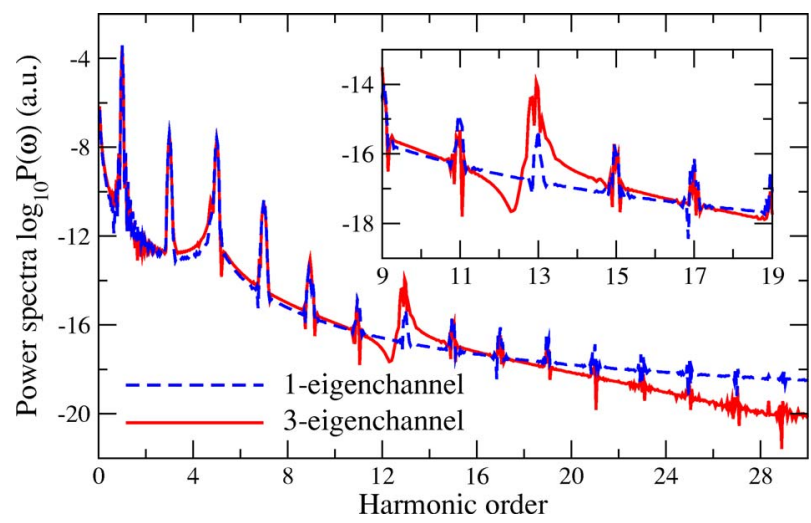

FIG. 8. (Color online) Comparison of the helium HHG by using different number of adiabatic eigenchannels. The laser pulse has a wavelength of $248.6 \mathrm{~nm}$, peak intensity of $I_{0}=1 \times 10^{14} \mathrm{~W} / \mathrm{cm}^{2}$ and a time duration of $\tau=30$ optical cycles. the electron correlation effect. Although the harmonic strengths are smaller, higher harmonics well beyond the fifth harmonic are noticeable from the full-dimensional twoelectron calculations. Note that in the conventional SAE model [13], the electron correlation effect has been completely neglected and no coupling exists between the response of the two electrons. The lack of higher harmonics in the SAE power spectra indicates that the appearance of harmonics with the orders larger than 5 should be attributed to the effect of electron correlation. Similarly, for the higher laser intensity case (Fig. 7), high harmonics beyond the 11th harmonic are also absent in the SAE spectra. The difference between the HHG power spectra of the ab initio two-electron and SAE calculations has been also noticed by the recent SIC-TDDFT calculation [14], including the electron correlation. Thus the significant effect of the electron correlation on the HHG dynamics and spectra can be clearly established.

\section{CONCLUSION AND OUTLOOK}

In summary, we have presented a nonperturbative $a b$ initio time-dependent hyperspherical coordinates approach for the exploration of the correlated quantum dynamics and the emitted HHG power spectra of the two-electron atomic systems in intense laser pulses. Our TDGPS approach developed in hyperspherical coordinates is shown to be capable of providing accurate time-dependent wave function and HHG power spectra with the use of only a modest number of grid points. The effects of the low-lying states and the doubly excited states on the HHG power spectra have been identified. The substantial difference between the results from the $a b$ initio two-electron and SAE calculations establishes the significance of the effect of the electron correlation. Extension of the present work beyond the adiabatic hyperspherical coordinates approach for the treatment of the double ionization process is in progress.

\section{ACKNOWLEDGMENTS}

This work was supported by the U.S. Department of Energy, Chemical Sciences, Geosciences and Biosciences Division of the Office of Basic Energy Sciences, and National Science Foundation. We acknowledge the support of the Origin 2000 supercomputer time provided by the Kansas Center for Advanced Scientific Computing.
[1] T. Brabec and F. Krausz, Rev. Mod. Phys. 72, 545 (2000).

[2] P. Salieres et al., Adv. At., Mol., Opt. Phys. 41, 83 (1999).

[3] Z. Chang H. Wang, M. M. Murnane, and H. C. Kapteyn, Phys. Rev. Lett. 79, 2967 (1997).

[4] Ch. Spielmann et al., Science 278, 661 (1997).

[5] M. Schnürer et al., Phys. Rev. Lett. 80, 3236 (1998).

[6] I. P. Christov, M. M. Murnane, and H. C. Kapteyn, Phys. Rev. Lett. 78, 1251 (1997).

[7] M. Hentschel et al., Nature (London) 414, 509 (2001).

[8] R. Kienberger et al., Science 297, 11449 (2002).

[9] Th. Udem et al., Nature (London) 416, 233 (2002).

[10] S. T. Cundiff and J. Ye, Rev. Mod. Phys. 75, 325 (2003).
[11] C. Gohle et al., Nature (London) 436, 234 (2005).

[12] See, for example, J. S. Parker, et al., J. Phys. B 33, 1057 (2000).

[13] See, for example, J. L. Krause, K. J. Schafer, and K. C. Kulander, Phys. Rev. A 45, 4998 (1992).

[14] X. M. Tong and S. I. Chu, Phys. Rev. A 57, 452 (1998); Int. J. Quantum Chem. 69, 293 (1998); Phys. Rev. A 64, 013417 (2001).

[15] C. A. Ullrich and E. K. U. Gross, Comments At. Mol. Phys. 33, 211 (1997).

[16] S. I. Chu, J. Chem. Phys. 123, 062207 (2005).

[17] X. Chu and S. I. Chu, Phys. Rev. A 63, 023411 (2001); 64, 
063404 (2001); 70, 061402(R) (2004).

[18] X. M. Tong and S. I. Chu, Chem. Phys. 217, 119 (1997).

[19] J. Macek, J. Phys. B 1, 831 (1968).

[20] See, for example, C. D. Lin, Phys. Rep. 257, 1 (1995).

[21] G. Yao and S. I. Chu, Chem. Phys. Lett. 204, 381 (1993); J. Wang, S. I. Chu, and C. Laughlin, Phys. Rev. A 50, 3208 (1994).

[22] S. I. Chu and X. M. Tong, Chem. Phys. Lett. 294, 31 (1998); X. M. Tong and S. I. Chu, Phys. Rev. A 61, 031401(R) (2000).

[23] X. M. Tong and C. D. Lin, Phys. Rev. A 71, 033406 (2005).

[24] O. I. Tolstikhin, S. Watanabe, and M. Matsuzawa, J. Phys. B 29, L389 (1996).

[25] K. Burnett, V. C. Reed, J. Cooper, and P. L. Knight, Phys. Rev.
A 45, 3347 (1992).

[26] Atomic, Molecular, and Optical Physics Handbook, edited by G. W. F. Drake (AIP, New York, 1996).

[27] M. K. Chen, Phys. Rev. A 56, 4537 (1997).

[28] E. J. Heller, J. Chem. Phys. 68, 3891 (1978).

[29] K. Kondo, A. Sagisaka, T. Tamida, Y. Nabekawa, and S. Watanabe, Phys. Rev. A 48, R2531 (1993).

[30] P. B. Corkum, Phys. Rev. Lett. 71, 1994 (1993).

[31] K. C. Kulander et al., in Super-Intense Laser-Atom Physics, NATO ASI Ser. B Vol. 316, p. 8-9, edited by B. Piraux et al. (Plenum, New York, 1993).

[32] D. H. Glass and P. G. Burke, J. Phys. B 32, 407 (1999). 